\title{
Clinical efficacy of multiple prevention measures against infec- tion following anterior cruciate ligament reconstruction
}

\author{
Rui Wang, Bin Xu*, Lei Wu, Bo Li \\ Department of sports medicine and arthroscopy, the First Affiliated Hospital of Anhui Medical University, Hefei 230032, China
}

\begin{abstract}
Background: A postoperative infection is one serious complication of an anterior cruciate ligament (ACL) reconstruction. Multiple infection prevention measures have been used before and after surgery, and their clinical efficacy was evaluated in this study.

Methods: This study enrolled 54 patients admitted to our hospital from January 2015 to June 2017 who underwent arthroscopic ACL reconstructions with hamstring tendons. One day before the surgery, the bilateral thighs and skin were washed using a potassium permanganate solution before and after bathing, separately. Aseptic surgical films containing iodine and disposable arthroscopic waterproof sterile drapes were used in the surgery. In addition, all the instruments used during the tendon transplantation were soaked and rinsed in a saline solution containing vancomycin. The Lysholm Knee Scoring Scale, International Knee Documentation Committee (IKDC) knee evaluation, and Tegner Activity Scale were administered before the surgery, 3 and 6 months after the surgery, and at the last follow-up. The averages of the scores at different stages were calculated. All the patients received a magnetic resonance imaging examination 3 months after the surgery.

Results: The patients were followed-up for 6 to 18 months (average $=10 \pm 1.4$ ), and no infections or other complications were found among them. When compared with the preoperative scores, the Lysholm score, IKDC knee evaluation score, and Tegner score were all significantly increased after the operation.

Conclusion: With multiple prevention measures adopted, no postoperative infections occurred in the patients, and the knee function recovered well. This suggests the success of the anti-infective therapy following the ACL reconstructions.
\end{abstract}

Keywords: knee arthroscopy, anterior cruciate ligament reconstruction, complications, infection

\section{INTRODUCTION}

The anterior cruciate ligament (ACL) is located in the knee joint. It restricts the excessive displacement of the tibia and maintains the stability of the knee, allowing for the complicated and highly challenging movements of the lower limbs ${ }^{[1]}$. An ACL injury is one of the most common and serious sports injuries, in which ACL tearing leads to knee joint instability, which can result in severe dysfunction in cases of unsuitable treatment ${ }^{[2]}$. Often accompanied by other damage, an ACL injury should be diagnosed and treated timely and properly; otherwise, certain complications may occur. Surgery should be performed to reconstruct the ligament and its functions. Thus, ACL reconstructions have always been a key subject in the field of orthopedics and sports injuries ${ }^{[3]}$. The arthroscopic

${ }^{*}$ Corresponding author: Bin Xu

Mailing address: Department of sports medicine and arthroscopy, the First Affiliated Hospital of Anhui Medical University, Hefei 230032, China

Address: Jixi Road No.218, Hefei, Anhui, 230022, China

Email: youchen100@126.com

Received: 20 January 2018 Accepted: 20 March 2018 method was the earliest developed minimally invasive technology used in orthopedics, and it largely guarantees the diagnoses and treatment of joint diseases. With the improvements in arthroscopic and surgical instruments, the surgical duration has been clearly shortened, and the accuracy and pertinence of this treatment have increased ${ }^{[4]}$. An arthroscopic ACL reconstruction is the symbolic operation in the field of sports injuries and orthopedics, and the hamstring tendon method is the most widely used ${ }^{[5]}$. However, in the long term clinical applications of this surgery, postoperative knee infections are rare but serious complications, with an incidence rate of 0.7$4.2 \%{ }^{[6.7]}$. A knee infection usually results in difficult wound healing, a limited range of knee motion, articular cartilage damage, and even traumatic arthritis. Moreover, some patients may need to undergo second or multiple operations to remove the graft and the internal fixation devices ${ }^{[8]}$. Therefore, measures to prevent postoperative infections after ACL reconstructions are urgently needed.

Previous reports have suggested ways to prevent postoperative infections after an ACL reconstruction. 
These infections have been divided into three stages: preoperative, intraoperative, and postoperative ${ }^{[9]}$. Previous reports have suggested ways to prevent postoperative infections after an ACL reconstruction. These infections have been divided into three stages: preoperative, intraoperative, and postoperative ${ }^{[10]}$. The intraoperative infection pathway covers the surgical instruments, grafts, fixation, and surgical incision, and many measures have been taken to minimize the possibility of infection ${ }^{[11]}$. It is a clinical consensus that inflammation in other parts of the body can be transported via the blood stream and cause knee infections, so infections in other parts of the body should be avoided after surgery ${ }^{[12]}$. With regard to the abovementioned information, we took a series of measures to prevent infections after ACL reconstructions and evaluated their efficacy.

\section{MATERIAIS AND METHODS}

\section{Clinical data}

Fifty-four patients admitted to our hospital from January 2015 to June 2017 who underwent arthroscopic ACL reconstructions using the hamstring tendon method were enrolled in this study. Various measures were taken before and after the surgery to prevent infection, and the clinical data were retrospectively analyzed after a follow-up of 6-18 months (average $=10 \pm 1.4$ ). Overall, 36 males and 16 females were included in this study, and their ages ranged from 17-46 years old (average $=32.5 \pm 1.6$ ). This study was approved by the ethics committee of the First Affiliated Hospital of Anhui Medical University.

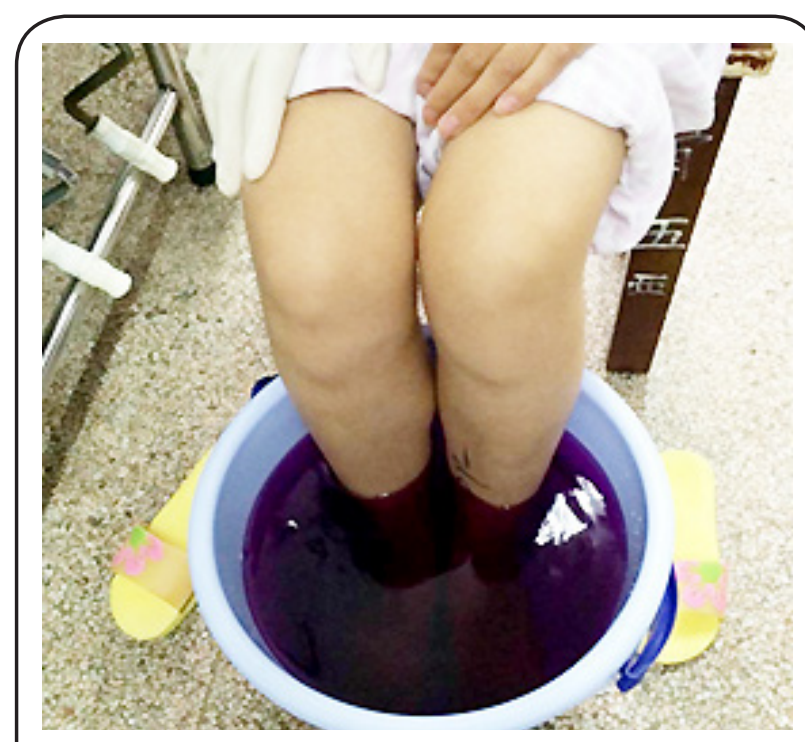

Figure 1 . The patients were asked to submerge their thighs in a potassium permanganate solution, with the knee joint line submerged under the solution, while washing the middle and lower thighs.

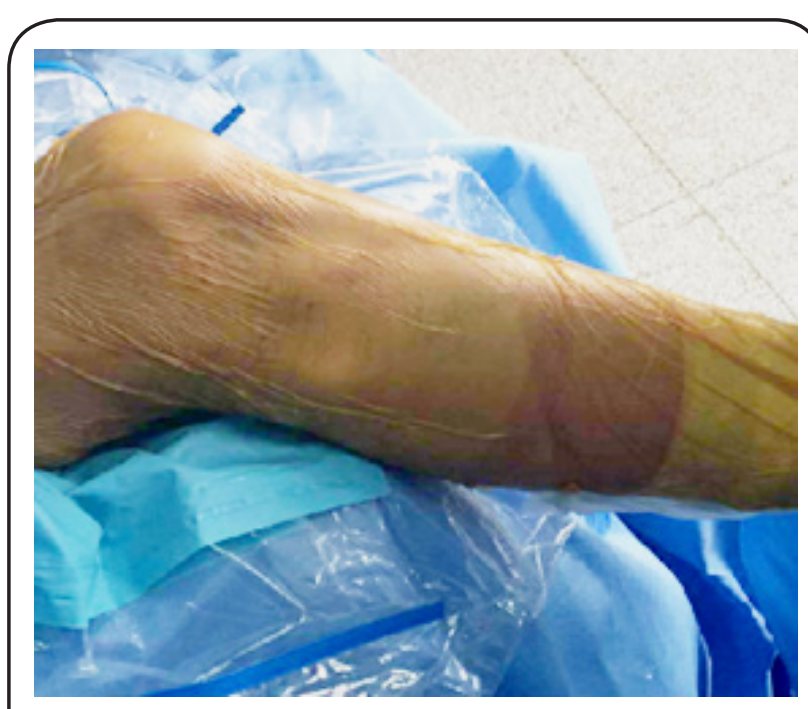

Figure 2. Disposable arthroscopic waterproof sterile drapes and aseptic surgical films containing iodine were used during the surgery.

Preoperative preparation

Before the surgery, each patient trimmed their toenails and fingernails, and they were required to bathe both lower extremities thoroughly from the groin to the feet. They were asked to change into clean underwear and patient gowns. In addition, the patients were asked to soak their bilateral thighs in a potassium permanganate solution, with the knee joint line submerged in the solution, while washing the middle and lower thighs (Figure 1).

Surgical procedures

Each patient underwent general anesthesia while lying in a horizontal position, with their knees sagging naturally at the beside, and a pneumatic tourniquet was placed below the hamstring root. Disposable

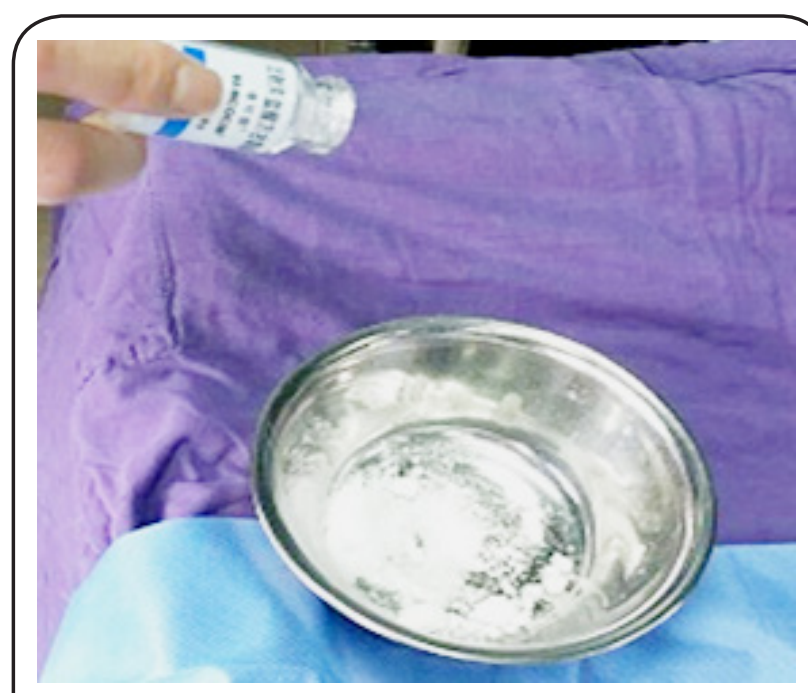

Figure 3. Using a saline solution, the vancomycin solution was prepared at a concentration of $5 \mathrm{mg} / \mathrm{ml}$. 


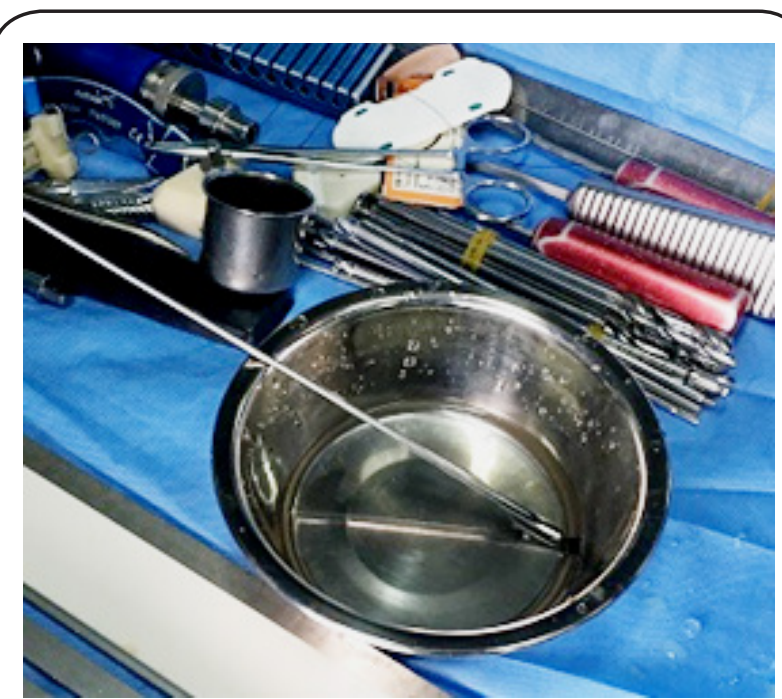

Figure 4. The tendon extractor and operating table were rinsed with a saline solution containing vancomycin.

arthroscopic waterproof sterile drapes (DP, USA) and aseptic surgical films containing iodine (3M6650, USA) were used during the surgery (Figure 2). Conventional anterior external and anterior internal arthroscopes were used to confirm the ACL injuries and any other combined injuries (meniscus or articular cartilage). These were dealt with according to the damage situation. An oblique incision of 2 cm was made below the ipsilateral tibial tubercle to expose the pes anserinus and the attachment point of the semitendinosus and gracilis at the tibia. The tendonextractor and operating table were rinsed with a saline solution containing vancomycin (Vianex SA, Athens, Greece) (Figures 3 and 4), and the tendon was removed. The tendon was bound and sutured using the No. 2 Ethibond braided wire). The draft line was reserved and folded 4 times, and the tendon was packed with operating gauze after the diameter detection to stand by until application. The viable stub end was cleaned and maintained, while the tibial and femoral bone tunnels were established. The skeletal remains and residue were thoroughly removed.
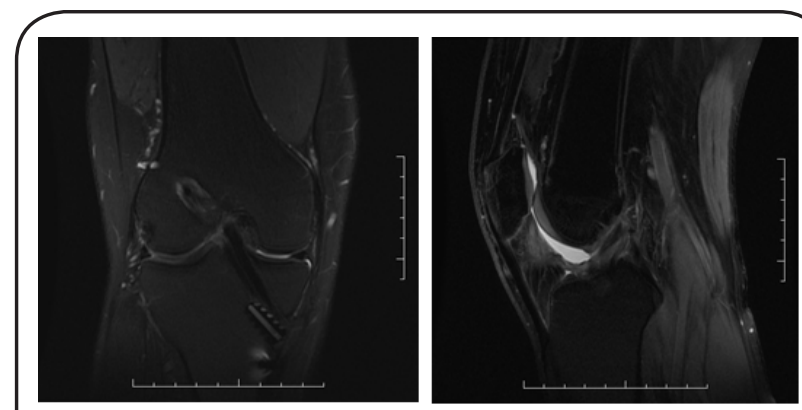

Figure 6. The magnetic resonance imaging examination performed 6 months after the surgery showed that the tendon in the bone tunnels of the tibia and femur grew well.
The proper size button plates (Endobutton; Smith \& Nephew, London, UK) were chosen according to the femur bone tunnel size, and the graft was placed inside the prefabricated loop. After rising it with vancomycin, the tendon was introduced to the femur bone tunnel by a leader wire, and it was fixed with a femoral-sided retroflex button, making sure that the bone tunnels of the tibia and femur were filled with the graft (Figure 5). Tension was applied to the lower end of the graft by stretching the knee, eliminating any impact or fixation loosening by bending and stretching it several times at an angle of $0-120^{\circ}$. Then, a posterior drawer test was conducted by pushing the proximal tibia at an angle of $30^{\circ}$. After the trailing end of the graft at the external bone tunnel of the tibia was frapped and maintained, the distal end of the graft was fixed with the proper bioabsorbable interference screw (Smith \& Nephew). With all the procedures completed, the articular cavity was washed thoroughly, and the redundant part of the graft was removed. Finally, the skin and subcutaneous

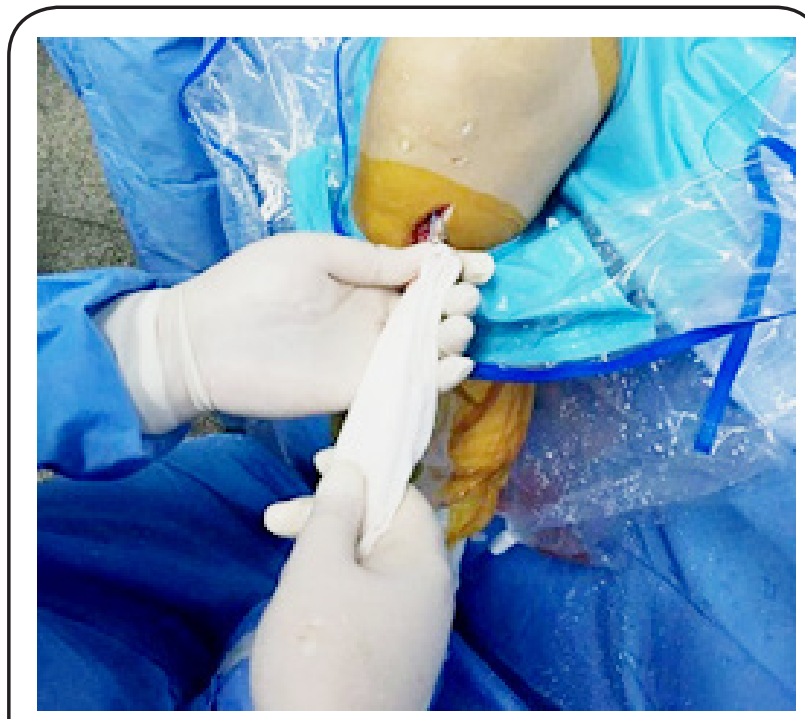

Figure 5. The tendon was rinsed in a vancomycin solution.

tissue were sutured.

Postoperative rehabilitation

A pressure dressing was applied to the affected knee with an elastic bandage after surgery, with cartridge tape support fixed at an angle of $0^{\circ}$. Some functional exercises, such as the isometric contraction of the quadriceps femoris, straight leg raising, and ankle pump exercises, were conducted $24 \mathrm{~h}$ after the operation, and the operation site was ice compressed. Three and 5 days after the operation, knee flexion exercises were conducted without weight bearing, and the flexion angle was gradually increased: 3-5 days after the intervention at an angle of $0-30^{\circ}$, 
7 days at $0-60^{\circ}, 2-3$ weeks at $0-90^{\circ}$, and 4 weeks at $0-120^{\circ}$. In the knee flexion restriction cases, combined continuous passive motion (CPM) could be used for the knee joint function, and partial weightbearing exercises could be carried out according to the recovery status of the myodynamia of the lower limbs. Eight and 12 weeks after the operation, normal walking without support was determined according to the recovery status of the myodynamia of the lower limbs. Moderate jogging was suggested 12 weeks after operation, and appropriate physical activity was suggested 4-6 months after the operation. A magnetic resonance imaging examination should be performed 6 months after the surgery, and the patient can participate in competitive sports one year later.

\section{Statistical analysis}

All the statistical data was analyzed using IBM SPSS Statistics for Windows version 21.0 (IBM Corp., Armonk, NY, USA). The measurement data were expressed as the mean \pm standard deviation (SD), and the pre- and postoperative comparisons were made using the Student's t-test. A p value of $<0.05$ was considered to be significant.

\section{RESUITS}

The patients were followed-up for 6 to 18 months (average $=10 \pm 1.4$ ). The incisions of these patients were healed at the first stage, and no infections or other complications, such as neurovascular injuries, cleavage fractures in the bone tunnels, and reconstructed ACL ruptures, were found among them. The judgment criteria for no infection were as follows: knee incision healed at the first stage without any inflammation, ulceration, or seepage, body temperature not exceeding $37.6^{\circ} \mathrm{C}$, and no obvious knee pain when slowly and passively flexed. The main knee movement range of all the patients was at the normal level (stretched at $-5^{\circ}-0^{\circ}$ and flexed at $0^{\circ}-120^{\circ}$ ) at the last follow-up. No traumatic arthritis clinical manifestations were found at the last follow-up, except for 2 cases of knee pain after activity and 5 cases with a small of amount of effusion in the articular cavity.

The magnetic resonance imaging examination performed 6 months after the surgery showed that the tendon in the bone tunnels of tibia and femur grew well, suggesting the success of the tendon reconstruction (Figure 6). Function evaluations were performed 3 and 6 months after the operation and at the last follow-up. When compared with the preoperative scores, the Lysholm Knee Scoring Scale, International Knee Documentation Committee (IKDC) knee evaluation, and Tegner Activity Scale were all significantly increased after the operation (Table 1).

\section{DISCUSSION}

An ACL reconstruction aims to recover the structural integrity of the ACL and remodel its biomechanical properties and physiological functions. After surgery, the ACL graft has to undergo a special "creeping substitution" process in order to remodel the fibrocytes ${ }^{[3]}$. No matter what surgical procedures and technologies are used, an infection following an ACL reconstruction is hard to eliminate, and it can result in many problems, including restricted functional rehabilitation, graft and articular cartilage damage, fixation loosening, and delayed healing of the incision[13.14]. Therefore, it is necessary to take certain measures, as far as possible, to prevent postoperative infections after ACL reconstructions.

Surgical area cleaning is the only requirement in traditional preoperative preparations. However, many studies of ACL reconstruction infections have indicated that Staphylococcus aureus and Staphylococcus epidermidis are the main pathogenic bacteria found in the synovial fluid and incision secretions, followed by Acinetobacter spp., Bacillus subtilis, and Pseudomonas aeruginosa, which are related with hospital-acquired infections ${ }^{[15,16]}$. In addition, fungal infections have been reported, which result mainly from tinea corporis and cruris and dermatophytosis ${ }^{[17]}$. These findings remind us that expanding the scope of cleaning, sterilization, and disinfection with a potassium permanganate solution may reduce the risk of postoperative infections, especially for skin with tattoos, hairy skin, and scarring skin ${ }^{[18]}$.

It has been reported that postoperative infections are more likely to occur when using the hamstring tendon method when compared with the allogenic tendon graft and artificial ligament methods ${ }^{[19]}$. This may be because of the universal use of hamstring tendons in ACL reconstructions. The use and repeated sterilization of the tendon interceptor and tendon plate increase the probability of introducing bacteria ${ }^{[20]}$. The increased soft tissue dissection area when obtaining the tendon is also an infection susceptibility factor. It has been proven that strict sterilization of the surgical area, the use of waterproof sterile drapes, high pressure steam sterilization of the surgical instruments, and a decrease in doctors and intraoperative personnel reduce the amount of bacteria in the air. In addition, reducing the continuous operation frequency and scheduling daytime surgery could effectively reduce the risk of infection ${ }^{[21]}$. Moreover, the aseptic surgical films containing iodine used during our operation also contributed to reducing skin exposure and diminishing 
the movement of the bacteria that likely exist in the folliculus pili.

The use of a saline solution containing vancomycin ( 5 $\mathrm{mg} / \mathrm{ml}$ ) in the ACL reconstruction to infiltrate the graft and implant achieved satisfactory results in the clinical application, even completely eradicating postoperative infections ${ }^{[22]}$. One interpretation may be that the transplanted tendon loses its blood supplement after interception, and the intravenous injection of antibiotics 30 minutes before the surgery just reduces the infection rate with regard to the receptor, while a donor tendon graft cannot be treated with antibiotics, offering the possible attachment of staphylococcus. The vancomycin-infiltrated graft perfectly resolves this problem by gradually delivering the drug and isolating the infection ${ }^{[23]}$. In our whole process of graft harvesting, suturing, and reimplantation, the vancomycin functioned as an antibiotic barrier to obstruct the bacteria.

Taken together, even though it is difficult to completely eradicate the postoperative infection risk after an ACL reconstruction, the comprehensive application of various preventative measures achieved a satisfactory outcome in our study. Certain complications were avoided, and postoperatively, the function recovered rapidly. However, a larger number of patients in the future may enhance the reliability of the study.

\section{ACKNOWLEDGEMENTS}

This study was supported by the grants from the Scientific and Technological Research Projects of "the application research of minimally invasive arthroscopic technology in diagnosis and treatment of diseases with unstable knee" (No. 1501041145)

\section{CONFLICT OF INTEREST}

The authors declare that there are no conflicts of interest with regard to this research.

\section{REFERENCES}

1. Bates, N. A., Myer, G. D., Shearn, J. T., and Hewett, T. E. (2015) Anterior cruciate ligament biomechanics during robotic and mechanical simulations of physiologic and clinical motion tasks: a systematic review and meta-analysis. Clin Biomech (Bristol, Avon) 30, 1-13

2. Failla, M. J., Arundale, A. J., Logerstedt, D. S., and Snyder-Mackler, L. (2015) Controversies in knee rehabilitation: anterior cruciate ligament injury. Clin Sports Med 34, 301-312

3. Kaeding, C. C., Pedroza, A. D., Reinke, E. K., Huston, L. J., Hewett, T. E., Flanigan, D. C., Group, M. K., and Spindler, K. P. (2017) Change in Anterior Cruciate
Ligament Graft Choice and Outcomes Over Time. Arthroscopy 33, 2007-2014

4. Martimbianco, A. L., Gomes da Silva, B. N., de Carvalho, A. P., Silva, V., Torloni, M. R., and Peccin, M. S. (2014) Effectiveness and safety of cryotherapy after arthroscopic anterior cruciate ligament reconstruction. A systematic review of the literature. Phys Ther Sport 15, 261-268

5. Zoran, Z., Ivan, V., Egon, B., Dubravka, B., Vjekoslav, W., and Vjekoslav, K. (2015) Knee stability after arthroscopic anterior cruciate ligament reconstruction using the middle third of the patellar ligament and quadrupled hamstring tendons grafts - A two-year follow-up. Injury 46 Suppl 6, S91-95

6. Bostrom Windhamre, H., Mikkelsen, C., Forssblad, M., and Willberg, L. (2014) Postoperative septic arthritis after anterior cruciate ligament reconstruction: does it affect the outcome? A retrospective controlled study. Arthroscopy 30, 1100-1109

7. Wylie, J. D., Marchand, L. S., and Burks, R. T. (2017) Etiologic Factors That Lead to Failure After Primary Anterior Cruciate Ligament Surgery. Clin Sports Med 36, 155-172

8. Kim, S. J., Postigo, R., Koo, S., and Kim, J. H. (2014) Infection after arthroscopic anterior cruciate ligament reconstruction. Orthopedics 37, 477-484

9. Kim, H. J., Lee, H. J., Lee, J. C., Min, S. G., and Kyung, H. S. (2017) Evaluation of Infection after Anterior Cruciate Ligament Reconstruction during a Short Period. Knee Surg Relat Res 29, 45-51

10. Whitehead, T. S. (2013) Failure of anterior cruciate ligament reconstruction. Clin Sports Med 32, 177204

11. Brophy, R. H., Wright, R. W., Huston, L. J., Nwosu, S. K., Group, M. K., and Spindler, K. P. (2015) Factors associated with infection following anterior cruciate ligament reconstruction. J Bone Joint Surg Am 97, 450-454

12. Ng, S. W., and Yee Han, D. L. (2015) Lessons learnt from an atypical mycobacterium infection postanterior cruciate ligament reconstruction. Clin Orthop Surg 7, 135-139

13. Gobbi, A., Karnatzikos, G., Chaurasia, S., Abhishek, M., Bulgherhoni, E., and Lane, J. (2016) Postoperative Infection After Anterior Cruciate Ligament Reconstruction. Sports Health 8, 187189

14. Greenberg, D. D., Robertson, M., Vallurupalli, S., White, R. A., and Allen, W. C. (2010) Allograft compared with autograft infection rates in primary anterior cruciate ligament reconstruction. J Bone 
Joint Surg Am 92, 2402-2408

15. Perez-Prieto, D., Trampuz, A., Torres-Claramunt, R., Eugenia Portillo, M., Puig-Verdie, L., and Monllau, J. C. (2017) Infections after Anterior Cruciate Ligament Reconstruction: Which Antibiotic after Arthroscopic Debridement? J Knee Surg 30, 309313

16. Pola, E., Logroscino, G., De Santis, V., Canducci, F, Delcogliano, A., and Gasbarrini, A. (2003) Onset of Berger disease after Staphylococcus aureus infection: septic arthritis after anterior cruciate ligament reconstruction. Arthroscopy 19, E29

17. Sun, L., Zhang, L., Wang, K., Wang, W., and Tian, M. (2012) Fungal osteomyelitis after arthroscopic anterior cruciate ligament reconstruction: a case report with review of the literature. Knee 19, 728731

18. Ibrulj-Prohic, D. (1989) [Postoperative treatment as an important factor in the prevention of adverse effects after dermabrasion of acne scars]. Med Arh 43, 23-26

19. Alomar, A. Z., Alfayez, S. M., and Somily, A. M. (2017) Hamstring autografts are associated with a high rate of contamination in anterior cruciate ligament reconstruction. Knee Surg Sports Traumatol
Arthrosc

20. Badran, M. A., and Moemen, D. M. (2016) Hamstring graft bacterial contamination during anterior cruciate ligament reconstruction: clinical and microbiological study. Int Orthop 40, 18991903

21. Murphy, M. V., Du, D. T., Hua, W., Cortez, K. J., Butler, M. G., Davis, R. L., DeCoster, T. A., Johnson, L., Li, L., Nakasato, C., Nordin, J. D., Ramesh, M., Schum, M., Von Worley, A., Zinderman, C., Platt, R., and Klompas, M. (2016) Risk Factors for Surgical Site Infections Following Anterior Cruciate Ligament Reconstruction. Infect Control Hosp Epidemiol 37, 827-833

22. Phegan, M., Grayson, J. E., and Vertullo, C. J. (2016) No infections in 1300 anterior cruciate ligament reconstructions with vancomycin pre-soaking of hamstring grafts. Knee Surg Sports Traumatol Arthrosc 24, 2729-2735

23. Schollin-Borg, M., Michaelsson, K., and Rahme, H. (2 003) Presentation, outcome, and cause of septic arthritis after anterior cruciate ligament reconstruction: a case control study. Arthroscopy 19, 941-947 